\title{
Dynamics of Flapping Micro-Aerial Vehicles
}

\author{
T.M. Yang and F.Y. Hsiao
}

\begin{abstract}
The dynamics of flapping wing micro aerial vehicles (MAVs) is studied in this paper. The MEMS Laboratory in Tamkang University has been developing flapping-wing MAVs for several years. Based on the developed flapping-wing MAVs we study its dynamics and compare our results with flight test data. Although several papers have discussed similar topics previously, using our flight test data we demonstrate the validity of the assumptions and derivations. We also propose a claim that links the average aerodynamical forces to the wind tunnel test data, so that a flapping MAV can be analyzed with the same methodology as what we have done to a fixed-wing aircraft. Flight test data and numerical simulations are also provided to demonstrate the validity of our derivation.
\end{abstract}

\section{INTRODUCTION}

Flight in flapping is a very efficient way to transport a unit of mass over a unit of distance, even thouth it requires extremely high power output[5]. For this reason, it is an interesting field and a new generation technology for the flight configuration. There are two kinds of flight configuration that is investigated in the literatures on natural flapping flight: Bird-like flight and Insect-like flight. The focus of this paper is on bird-like flight. The bird-like aerial robot we are investigating is developed by the TKU MEMS $\mathrm{LAB}$ in the recent years.

The TKU MEMS Laboratory has been developing birdlike flapping MAVs for several years. Figure 1 demonstrates the most recent prototype, "Golden Snitch", which is a 7-gram-weight and 20-cm-wingspan aircraft including the fuselage, flapping wings, tail wing, battery, motor and a set of gear system. The flapping wing is driven by a motor with a four-bar linkage system. By adjusting the lengths of the four bars, various stroke angles can be designed. In Golden Snitch the stroke angle is designed around $53^{\circ}$. [2].

The aerodynamics performance in flapping animals consists of delayed stall, rotational circulation and wake capture [10]. These phenomenon and their functions can be explained by experiments and theories. However, complete and exact analysis of the flapping flight is not available because of the aerodynamic and mechanical complexity. As a result, In Ref. [4] Kim developed a smart flapping wing with a macro-fiber compositers (MFC) actuator to mimic the flying mechanism to measure the aerodynamic forces of flapping devices in wind tunnel test. Furthermore, In Ref. [7] Rakotomamonjy investigates the optimization of the flapping

Tse-Ming Yang is with graduate student of Aerospace Engineering, Tamkang University, Tamsui 251, Taiwan, ROC 492370365es92.tku.edu.tw

Fu-Yuen Hsiao is with faculty of Aerospace Engineering,Tamkang University, Tamsui 251, Taiwan, ROC fyhsiao@mail.tku.edu.tw

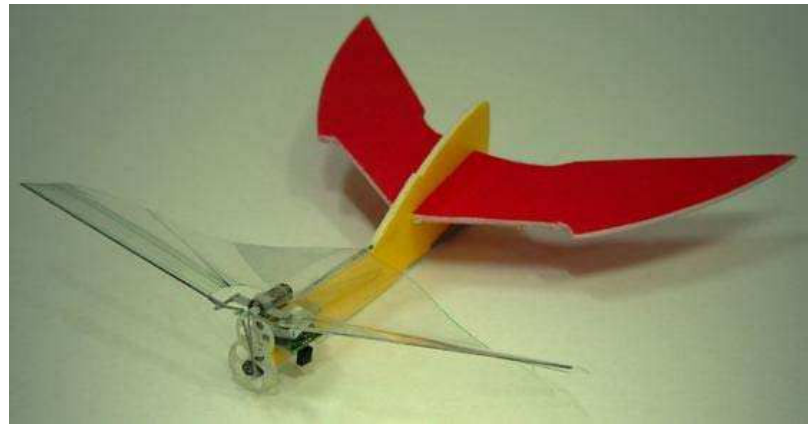

Fig. 1. The flapping MAV developed by the TKU MEMS Lab.[11]

kinematics of the wing. In the full dynamic model of flapping MAV, Zaeem built a longitudinal flight dynamics with timeaverage theory [3], but only in 2-dimension space. In this paper, we intend to develop the three-dimensional model which will then be compared with the real trajectory.

In this research, we investigate the dynamic model of flapping MAV. Starting from Newton's second law we develop the equations of motion of our flapping-wing robot. Due to the fast flapping frequency compared with the translational and rotational rates, the average lift and thrust forces over each flapping period are applied to this model. Numerical simulations are also provided to examine the validity of our model and selected parameters.

\section{Dynamic Model}

\section{A. Equations of Motion}

a)

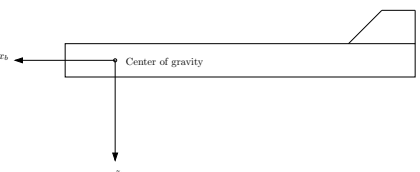

Fig. 2. A cartoon showing the definition of the body-fixed frame.

Before the formulation of equations of motion (EOM) a body-fixed frame is defined in Fig. 2 . The $x_{b}$-axis points forward along the axis of the fuselage in the MAV's plane of symmetry. The $y_{b}$-axis is normal to the plane of symmetry pointing in the direction of the right wing. The $z_{b}$-axis then points downward in the MAV plane of symmetry, completing the right-handed Cartesian system. In addition, the coordinates in the inertial frame are denoted as $\left(x_{f}, y_{f}, z_{f}\right)$ in this paper. The transformation between these two frames can be 
accomplished by a rotational matrix $\mathbf{R}$, satisfying

$$
\begin{aligned}
\mathbf{V}_{f} & =\mathbf{R V}_{b} \\
\dot{\mathbf{R}} & =\mathbf{R} \tilde{\omega}
\end{aligned}
$$

where $\mathbf{V}_{f}$ and $\mathbf{V}_{b}$ denote any vectors in the inertial and body-fixed frames, respectively. $\tilde{\omega}$ is the cross product operator of the angular velocity $\vec{\omega}=\left(\omega_{x}, \omega_{y}, \omega_{z}\right)$ [8].

The equations of motion of the flapping wing MAV can be obtained by applying Newton's second laws, given by

$$
\begin{aligned}
\sum \mathbf{F} & =m \frac{d}{d t} \mathbf{V}+\omega \times(m \mathbf{V}) \\
\sum \mathbf{M} & =\mathbf{I} \frac{d}{d t} \vec{\omega}+\vec{\omega} \times(\mathbf{I} \vec{\omega})
\end{aligned}
$$

where I denotes the inertia tensor. The external forces includes the weight of the vehicle, aerodynamical forces by flapping wing, horizontal tail wing, and vertical tail. Those forces also generates moments about the center of gravity (CG). We should notice that Eqs. (3) and (4) are the EOM described in the body-fixed frame, where the velocity in the body-fixed frame has components $(u, v, w)$, and the angular velocity has components ( $p, q, r)$. The expansion of Eqs. (3) and (4) can be found in [6].

\section{B. Averaging Theory and Formulation of Forces}

1) Applicability of Averaging Theory: Due to the periodic motion of the flapping wings, the averaging theory is usually applied to analyze the dynamics of a flapping wing robot, such as in Refs. [3] and [8]. The averaging theory is applicable based on the assumption that the wing is much lighter than the the body. As a result, the flapping wing slightly affects the vertical motion of the vehicle.

Even though the assumption sounds reasonable, it seems that no flight test data has been shown in the literature. In [3] a control law is designed based on this assumption while in [8] a ground-based experiment has been designed to investigate the controllability of a biomimic MAV.

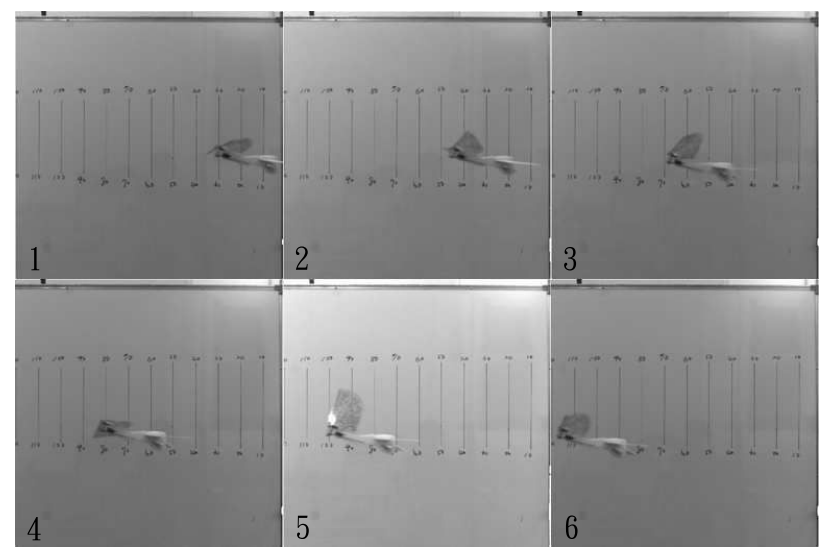

Fig. 3. The cruise flight of Golden Snitch catched by high speed CCD camera.[12]

Our Golden Snitch, however, verifies the validity of this assumption. As we can see in Fig. 3, Golden Snitch flies forward in a velocity of $\sim 3 \mathrm{~m} / \mathrm{s}$, but the fuselage still remains at an almost fixed height when the wings are flapping.
2) Averaged Force and Advance Ratio: In addition to the applicability of averaging theory, there was still one thing unclear before. Although the averaging theory was assumed to be applicable to the dynamical analysis of a flapping wing robot, the researchers in control field were still not clear about the formulation of the averaged lift and thrust forces. Accordingly, dynamics and control scientists usually simulated the lift and thrust force with a simple function, such as a periodic triangular wave.

On the other hand, the researchers in aerodynamics field always formulate the lift and thrust forces generated by a flapping wing as a function of the advance ratio, $J$, defined as

$$
J=\frac{U}{2 b f \Phi}
$$

where $\Phi, f$, and $\mathrm{b}$ are stroke angle, flapping frequency, and wing semi-span, respectively. Typically, unsteady-state flight has advance ratio $J$ less than 1 . Low advance ratio $J$ is an indication that these flyers must flap their wings at high speed compared to the speed of their flights in order to stay aloft. Therefore, the regime of $J<1$ is dominated by unsteady-state flight. On the other hand, for $J \gg 1$, the flight regime becomes quasi-steady and approaches steady-state. For example, a fixed-wing airplane operates in the regime of $J$ near infinite because the wings' flapping frequency is zero. The lift and thrust forces can be expressed as functions of $J$ [2]

$$
\begin{aligned}
F_{\text {lift }} & =\frac{1}{2} \rho U^{2} S C_{L}(J) \\
F_{\text {thrust }} & =\frac{1}{2} \rho U^{2} S C_{T}(J)
\end{aligned}
$$

where $C_{L}(J)$ and $C_{T}(J)$, as functions of $J$, denote the lift coefficient and thrust coefficient, respectively.

Here we claim that the forces calculated from the lift or thrust coefficient as a function of $J$ can be treated as the averaged force. A simple proof goes below. Consider
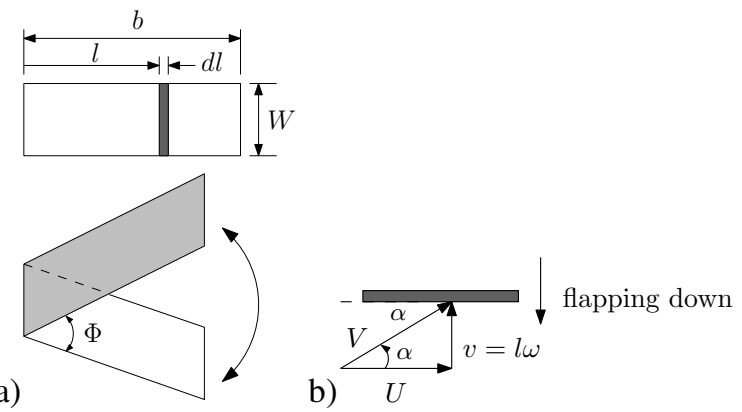

Fig. 4. A cartoon showing the definition of wing parameters.

a very thing rectangular wing, as shown in Fig. 4(a), with length $b$, width $W$, stroke angle $\Phi$, and flapping frequency $\omega=2 \pi f$. Assume the setting angle is zero so that the angle of attack (AOA) is determined by the attacking angle of the incoming air stream completely. Consider a small area element on the wing, whose flapping motion is shown in 
Fig. 4(b). According to aerodynamics theory, the lift force generated by this element is formulated as

$$
d F=\frac{1}{2} \rho V^{2} C_{L}(\alpha) d A
$$

where $V^{2}=U^{2}+(l \omega)^{2}, d A=W d l$, and

$$
\alpha=\arctan \left(\frac{l \omega}{U}\right)
$$

To simplify the notation we define $l / b=\gamma$. Introducing the advance ratio we obtain

$$
l \omega=\frac{\gamma \pi}{J \Phi} U
$$

As a result, Eq. (8) can be reformulated as

$$
\begin{aligned}
d F & =\frac{1}{2} \rho V^{2} C_{L}(\alpha) d S \\
& =\frac{1}{2} \rho U^{2} W b\left[1+\left(\frac{\pi}{J \Phi}\right)^{2} \gamma^{2}\right] C_{L}(\alpha) d \gamma \\
& =\frac{1}{2} \rho U^{2} S\left[1+\left(\frac{\pi}{J \Phi}\right)^{2} \gamma^{2}\right] C_{L}(\alpha) d \gamma
\end{aligned}
$$

where $S=W b$ is the total area of the wing, and $\alpha=$ $\alpha(J, \gamma)$. Consider the average force during the downstroke during time interval $T_{d}$, given by

$$
\begin{aligned}
\bar{F} & =\frac{1}{T_{d}} \int_{0}^{T_{d}} F(t) d t \\
& =\frac{1}{T_{d}} \int_{0}^{T_{d}} \int_{0}^{F} d F d t \\
& =\frac{\rho U^{2} S}{2 T_{d}} \int_{0}^{T_{d}} \int_{0}^{1}\left[1+\left(\frac{\pi}{J \Phi}\right)^{2} \gamma^{2}\right] C_{L}(\alpha) d \gamma d t
\end{aligned}
$$

Since the integrant is not an explicit function of time, we can integrate with respect of time first and null out $T_{d}$. Therefore,

$$
\bar{F}=\frac{\rho U^{2} S}{2} \int_{0}^{1}\left[1+\left(\frac{\pi}{J \Phi}\right)^{2} \gamma^{2}\right] C_{L}(\alpha(J, \gamma)) d \gamma
$$

Define

$$
C_{L}^{\prime}(J)=\int_{0}^{1}\left[1+\left(\frac{\pi}{J \Phi}\right)^{2} \gamma^{2}\right] C_{L}(\alpha(J, \gamma)) d \gamma
$$

We obtain that

$$
\bar{F}_{d}=\frac{1}{2} \rho U^{2} S C_{L_{d}}^{\prime}(J)
$$

where the subscript $d$ denotes downstroke. Similarly, the average force during the upstroke is given by

$$
\bar{F}_{u}=\frac{1}{2} \rho U^{2} S C_{L_{u}}^{\prime}(J)
$$

As a result, the average force generated during a complete flapping is given by

$$
\begin{aligned}
\bar{F} & =\bar{F}_{d}+\bar{F}_{u} \\
& =\frac{1}{2} \rho U^{2} S C_{L_{d}}^{\prime}(J)+\frac{1}{2} \rho U^{2} S C_{L_{u}}^{\prime}(J) \\
& =\frac{1}{2} \rho U^{2} S C_{L}^{\prime}(J)
\end{aligned}
$$

where $C_{L}^{\prime}(J)=C_{L_{d}}^{\prime}(J)+C_{L_{u}}^{\prime}(J)$. We can see that the average force has the same formulation as Eqs. (6) and (7).

We would admit that this is not a rigorous proof because many aerodynamics factors are not considered, such as the stability of the air flow, the flexibility of the wing and so on. However, at least this proof gives a qualitative link between the average force used in the dynamics field and the most common way to formulate flapping lift and trust forces in the aerodynamics field. In other words, if we have the lift and thrust coefficient curves at hand, which are usually easy to obtain in aerodynamics journals, we can simply apply the same methodology of analyzing a fixed-wing vehicle to the analysis of a flapping-wing robot.

\section{Formulation of Forces and Moments}

Having shown that the average forces over one flapping period can be calculated by using Eqs. (6) and (7), which is independent of time, we conclude that the methodology to analyze a fixed wing vehicle can be applied to the flapping wing vehicle. There are only two differences. First of all, the force coefficients $C_{L}$ and $C_{T}$ are no longer functions of angle of attack only, but also functions of advance ratio. Second, when applied to analyze the dynamics of the whole vehicle, we don't use angle of attack since it is not rigorously defined in flapping motion. Instead, the set angle and stroke angle are introduced.

Figures. 5(a) and (b) provide the distribution of aerodynamics forces on the wing. As a result, provided Eqs. (6) and (7) $F_{x_{b}}$ and $F_{z_{b}}$ can be obtained by considering the vector addition of the lift and thrust forces.

$$
\begin{aligned}
& F_{z_{b_{\text {wing }}}}=F_{\text {thrust }} \sin (\alpha)-F_{\text {lift }} \cos (\alpha) \\
& F_{x_{b_{\text {wing }}}}=F_{\text {thrust }} \cos (\alpha)+F_{\text {lift }} \sin (\alpha)
\end{aligned}
$$

where $\alpha$ is set angle of MAV.

a)

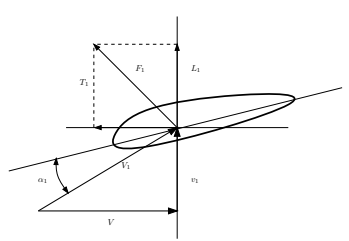

b)

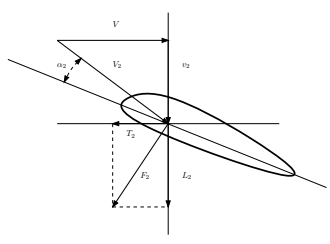

Fig. 5. The aerodynamic force distribution during a)downstroke. b) upstroke.

On the other hand, the moments exerted on the MAV can be obtained through summing up all individual moment and torque. All the necessary geometric parameters to calculate moments are shown in Fig. 6. In addition to the regular formulation of moments, one thing to remind again is that we have to consider the torque applied by the motor, $\tau=\tau_{\text {motor }}$ pointing along $+x_{b}$-axis because our motor spins clockwise. Applying the formulated forces and moments to Eqs. (3)-(4) we can solve for the position, velocity and attitude of the MAV. 


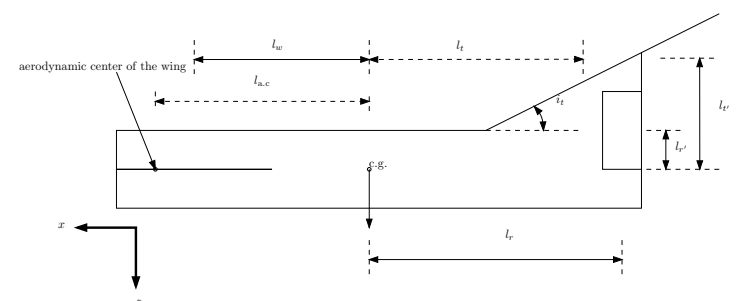

Fig. 6. A cartoon showing the geometric parameters of the fuselage.

\begin{tabular}{lrrrrr}
\hline Set angle & $10^{\circ}$ & $20^{\circ}$ & $30^{\circ}$ & $40^{\circ}$ & $50^{\circ}$ \\
\hline$a$ & 19.07 & 20.22 & 35.35 & 42.09 & 58.25 \\
$b$ & 5.471 & 4.174 & 4.851 & 4.823 & 6.107 \\
$c$ & 0.6914 & 1.181 & 1.404 & 2.051 & 2.346 \\
$a^{\prime}$ & 109.8 & 103.9 & 153.2 & 156.2 & 92.43 \\
$b^{\prime}$ & 7.878 & 8.168 & 11.05 & 10.58 & 9.154 \\
$c^{\prime}$ & 0.3139 & 0.1475 & 0.01054 & -0.5002 & -0.8389 \\
\hline
\end{tabular}

TABLE I

THE PARAMETERS IN FORCE COEFFICIENTS FOR A FLAPPING WING.[2]

\section{Coefficients of the Main Wing}

According to Ref. [1], the coefficient of lift and coefficient of thrust can be modeled as:

$$
\begin{gathered}
C_{L_{\text {wing }}}=a e^{-b J}+c \\
C_{T_{\text {wing }}}=a^{\prime} e^{-b^{\prime} J}+c^{\prime}
\end{gathered}
$$

For the TKU flapping MAV, those parameters are obtained through wind tunnel test, and list as a function of set angle in Tab. (I). With the lift and thrust coefficients, the forces can be obtained using Eqs. (6) and (7). According to the result from the proceeding section, the obtained forces will be the average ones over one flapping period. An example showing the variation of forces as a function of time is shown in Figs. 7 and 8.

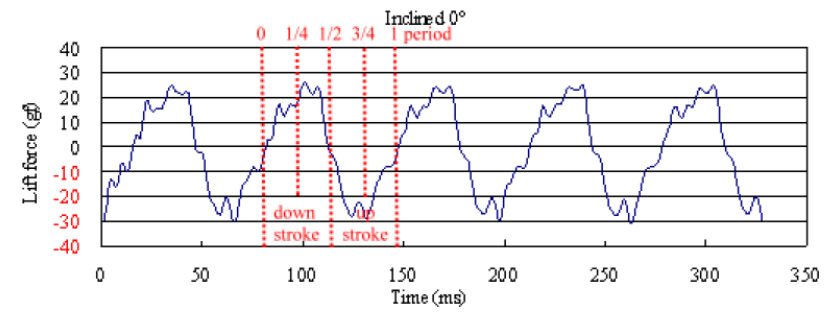

Fig. 7. The variation of lift force during a flapping period.[2]

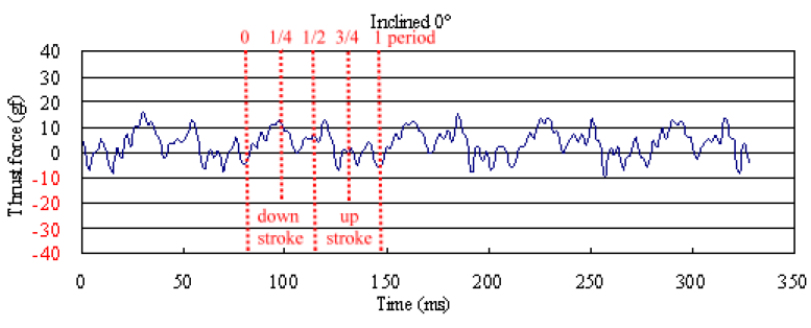

Fig. 8. The variation of thrust force during a flapping period.[2] a)

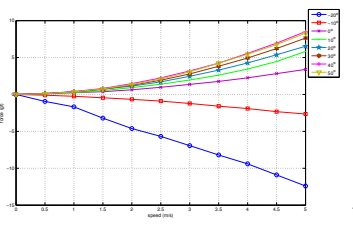

b)

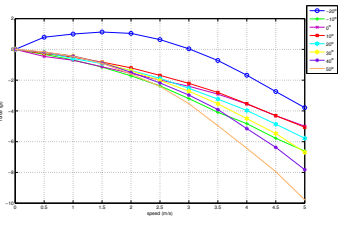

Fig. 9. The lift and drag performance of tail wing in wind tunnel test.

\begin{tabular}{lrrrrrrrr}
\hline AOA & $-20^{\circ}$ & $-10^{\circ}$ & $0^{\circ}$ & $10^{\circ}$ & $20^{\circ}$ & $30^{\circ}$ & $40^{\circ}$ & $50^{\circ}$ \\
\hline$C_{L_{\text {tail }}}$ & -1.242 & -0.2808 & 0.3571 & 0.5963 & 0.6881 & 0.8148 & 0.9025 & 0.8868 \\
$C_{D_{\text {tail }}}$ & -0.4868 & -0.695 & -0.4938 & -0.5229 & -0.5974 & -0.6971 & -0.8186 & -1.04 \\
\hline
\end{tabular}

TABLE II

THE PARAMETERS IN FORCE COEFFICIENTS FOR A TAIL WING.

\section{E. Coefficients of the Horizontal Wing}

In addition to the main wing, the tail wing is still to be considered. The tail-wing parameters of aerodynamic forces is obtained through wind tunnel test, shown in Figs. 9(a) and (b). The tail-wing angle of attack ranges from $-20^{\circ}$ to $50^{\circ}$ and wind speed ranges from $0 \mathrm{~m} / \mathrm{s}$ to $5 \mathrm{~m} / \mathrm{s}$.

According to aerodynamics, lift and drag coefficients are given by

$$
\begin{aligned}
C_{L_{\text {tail }}} & =\frac{2 L_{\text {tail }}}{\rho U^{2} S_{\text {tail }}} \\
C_{D_{\text {tail }}} & =\frac{2 D_{\text {tail }}}{\rho U^{2} S_{\text {tail }}}
\end{aligned}
$$

where $\rho$ is density of air, $S_{\text {tail }}$ is the area of the tail wing, $L_{\text {tail }}$ and $D_{\text {tail }}$ denote the lift and drag of the tail wing, respectively. For Golden Snitch, the area of tail wing is about $6013.715 \mathrm{~mm}^{2}$, and the density of air is about $1.23 \mathrm{~kg} / \mathrm{m}^{3}$. Hence,

$$
\begin{aligned}
C_{L_{\text {tail }}} U^{2} & =2.65 L_{\text {tail }} \\
C_{D_{\text {tail }}} U^{2} & =2.65 D_{\text {tail }}
\end{aligned}
$$

where the unit of $U$ and $L_{\text {tail }}\left(D_{\text {tail }}\right)$ are $\mathrm{m} / \mathrm{s}$ and gf, respectively.

\section{Flight Test and Numerical Simulation}

Having shown that the averaging theory is applicable to the flapping wing MAV, and the way to obtain the average forces from experiment data, we apply this result to the analysis of our Golden Snitch.

\section{A. Flight Test}

The Golden Snitch has been put into flight test and its flight duration is about 5 minutes. Some examples of the flight trajectory are provided in Figs. 10- 12 [2]. The only control applied to this vehicle is the spin rate of the motor, which controls the flapping frequency. Currently, there is no control of direction. The flight trajectory, however, is spiral. This is resulted from the torque generated by the motor due to the conservation of angular momentum. 


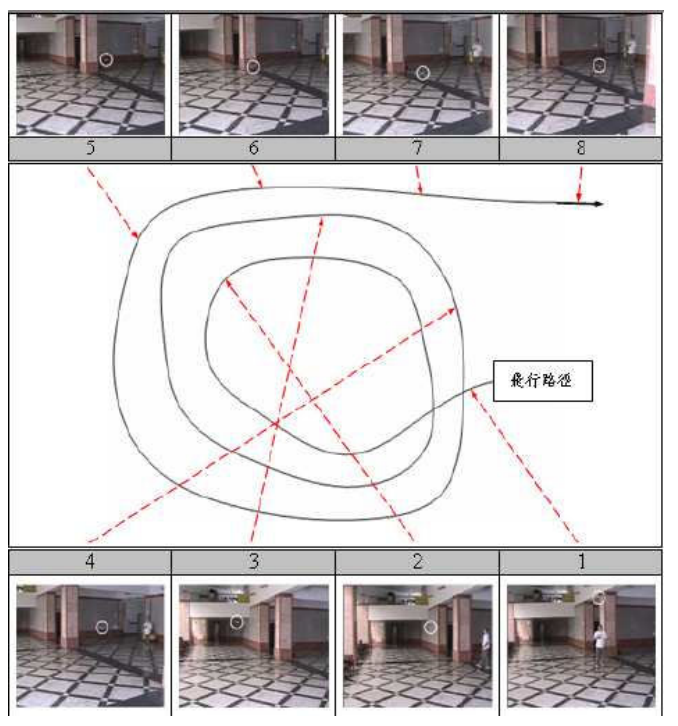

Fig. 10. An example of the flight test trajectory. [2]

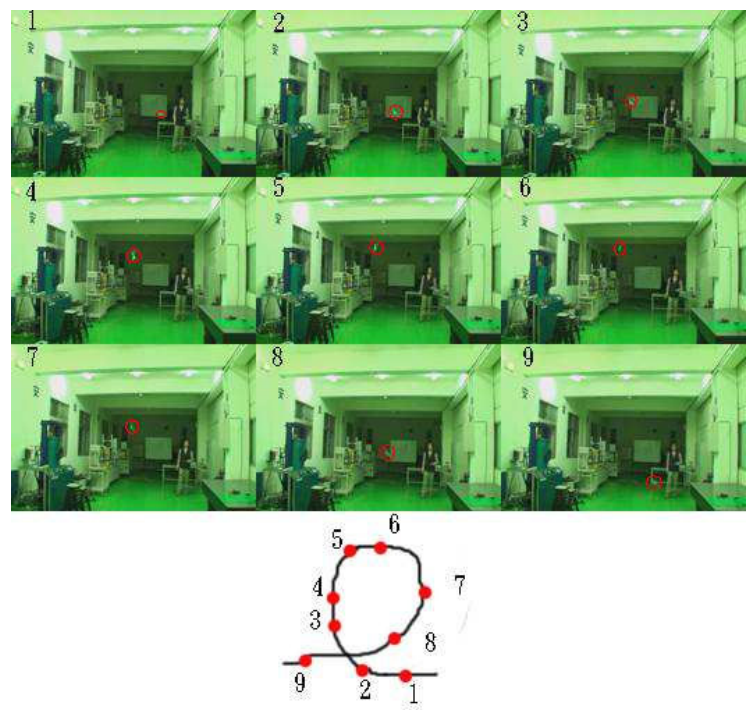

Fig. 11. An example of the flight test trajectory. [2]

\section{B. Numerical Simulation}

1) Attitude Equilibria at Cruise Flight: At cruise flight the MAV must be in the equilibria of its attitude. According to our model, we obtain that the pitch angle at cruise flight must be $12^{\circ}$. Examining Fig. 3 we realize that the Golden Snitch flies at the angle of $15^{\circ}$. This is encouraging since our prediction is quite close to the reality. However, this fact also implies that the predicted lift force at wing tail may be too large so that the pitch angle is smaller than the real one.

2) Simulated Flight Trajectories: Besides encouraging result in the match of derived and actual attitude equilibria, three dynamical cases are also simulated, provided in Figs. 13- 15. In the first case, shown in Fig. 13, we use the derived parameters from the previous chapter but neglect the torque made by the motor. The simulated flight trajectory forms a circle. In the second case we reduce the coefficients
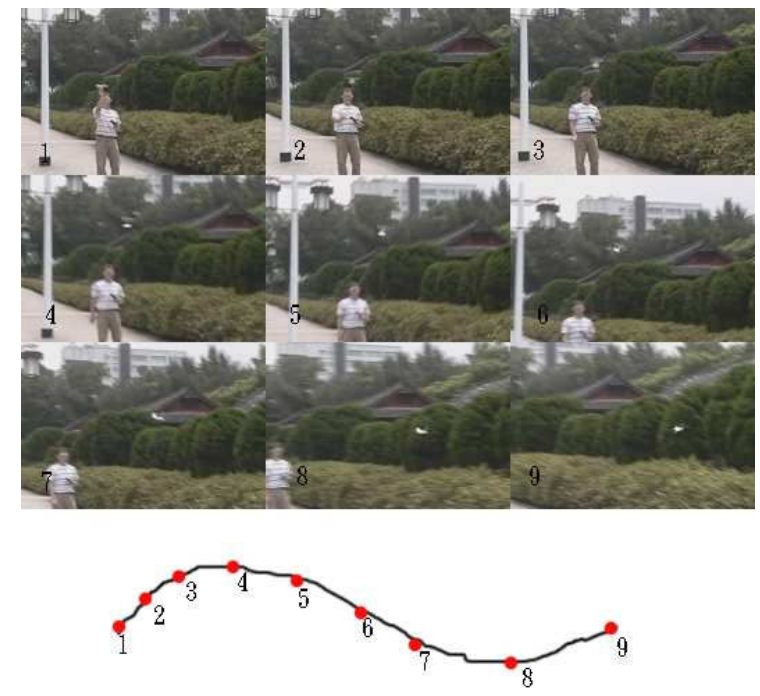

Fig. 12. An example of the flight test trajectory. [2]

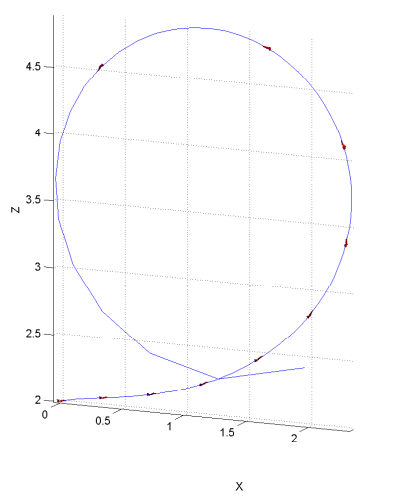

Fig. 13. The flight trajectory in case 1 .

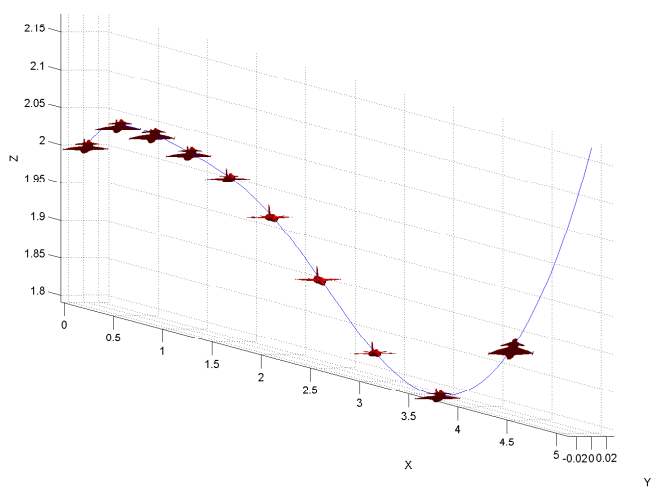

Fig. 14. The flight trajectory in case 2 . 


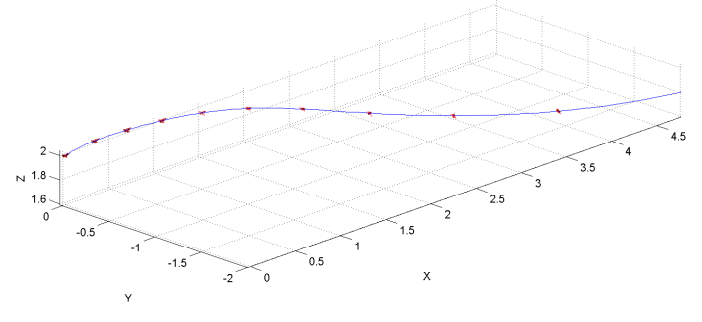

Fig. 15. The flight trajectory in case 3 .

of main wing by half. The flight trajectory is presented in Fig. 14. In the case 3, we take into consideration the torque generated by the motor and use treat other parameters the same as in case 2. The flight trajectory is shown in Fig. 15.

3) Discussion: In the simulation of case 1, the moment in $y_{b}$ direction seems too large and that makes MAVs do a circular motion. This simulation matches one case in flight test, given in Fig. 11. In the case 2 we reduce half of the lift and thrust coefficients. This time the MAV stays reasonable flight for longer time, but it still attempts to do a circular motion, too. It is obvious that this simulation resembles the case shown in Fig. 12. A reasonable explanation goes that the lift might be smaller outdoors since there exist disturbances. As a result, the simulation assumption in case 2 is also reasonable. In case 3 , we consider the torque generated by the motor, and it will induce the lateral motion. We can imagine that the trajectory must be spiral since the vehicle is lifting up and turning clockwise.

These cases demonstrate the creditability of the dynamic model of MAVs. According to the above simulations and records from flight tests, we successfully obtain different trajectories that also occurs in flight test.

\section{CONCLUSIONS}

In this paper we study the dynamics of flapping-wing MAVs. Our results are also compared with the flight test data, using the flapping-wing MAVs developed by TKU MEMS Lab. Starting from Newton's second law, we derive the equations of motion for the MAVs. By observing the cruise flight of our MAVs in the high speed CCD Camera, we show that flapping doesn't affect the vertical motion of the whole vehicle, implying that the averaging theory is applicable. We also analytically prove that the time-average forces (lift and thrust) have the same formulation as those in the conventional fixed wing, while the only difference is the coefficient of lift, which is a function of advance ratio and set angle instead of angle of attack. As a result, having the force coefficient curves from wind tunnel test data, we can simulate the dynamics immediately without assuming the time history of those aerodynamics forces. Numerical simulations are also provided in this paper. Our numerical simulations not only catch the trend of the flight test trajectory, but also match the cruise flight condition.

\section{ACKNOWLEDGEMENT}

The work described here is funded by the National Science Council through project NSC 97-2221-E-032-017. The authors wish to thank Dr. Lung-Jieh Yang and his research group for providing flight test and wind tunnel test data.

\section{REFERENCES}

[1] M.H. Dickinson, F.O. Lehmann, and S.P. Sane, "Wing rotation and the aerodynamic basis of insect flight," Science, Vol. 284, pp. 1954-1960, 1999

[2] C. K. Hsu, The Preliminary Design, Fabrication, and Testing of Flapping Micro Aerial Vehicles, Ph.D. Dissertation, Tamkang University, 2008

[3] Z.A. Khan, and S.K. Agrawal, "Control of Longitudinal Flight Dynamics of a Flapping-Wing Micro Air Vehicle Using Time-Averaged Model and Differential Flatness Based Controller," Proceedings of the 2007 American Control Conference, pp. 5284-5289, 2007

[4] Dae-Kwan Kim, and Jae-Hung Han, "Smart flapping wing using Macro-Fiber Composite actuators," Proceeding of Smart Structures and Materials, 2006

[5] U.M. Norberg, Vertebrate Flight: Mechanics, Physiology, Morphology, Ecology and Evolution, Springer-Verlag, 1990

[6] W.F. Phillips, Mechanics of Flight, John Wiley \& Sons, 2004

[7] T. Rakotomamonjy, M. Ouladsine, and T. Le Moing, "Modelization and Kinematics Optimization for a Flapping-Wing Microair Vehicle," Journal of Aircraft, Vol. 44, 2007

[8] L. Schenato, D. Campolo, and S. Sastry, "Controllability issuse in flapping flight for biomimic MAVs," Proceedings of the 42nd IEEE Conference on Decision and Control, pp. 6441-6447, 2003

[9] M. Sun, J. Wang and Y. Xiong, "Dynamic flight stability of hovering insects," Acta Mechanica Sinica, 2007

[10] J. Yan, R.J. Wood, S.S. Avadhanula, , R.S.M. Fearing, "Towards flapping wing control for a micromechanical flying insect," IEEE International Conference on Robotics and Automation, 2001

[11] Yang Lung-Jieh, Hsu Cheng-Kuei, Hsiao Fu-Yuen and Shen YungKang, "A Micro-Aerial-Vehicle(MAV) with Figure-of-Eight Flapping Induced by Flexible Wing Frames" 47th AIAA Aerospace Sciences Meeting, 2009, Orlando, Florida

[12] L.J. Yang, M.W. Kao, C.K. Hsu, C.W. Liao, I-C. Huang and P.T. Fang, "A biomimetic figure-of-eight flapping of micro aerial vehicles(MAVS) illuminated by LEDS," Asia-Pacific Conference on Transducers and Micro/Nanotechnology (APCOT 2008), 2008, Tainan, Taiwan 